\title{
THE SHAPE OF THE STRONGEST COLUMN AND SOME RELATED EXTREMAL EIGENVALUE PROBLEMS*
}

\author{
$\mathrm{BY}$
}

EARL R. BARNES

IBM Thomas J. Watson Research Center, Yorktown Heighls

\begin{abstract}
We determine the shape of the strongest column in the class of columns of length $l$, volume $V$, and having similar cross-sectional areas $A(x)$ satisfying $a \leq A(x)$ $\leq b$ where $a$ and $b$ are prescribed positive bounds. In the special case where there are no constraints on the areas of cross-sections the problem has been solved by Keller [1] and by Takjbakhsh and Keller [2]. These authors observed that the problem is equivalent to an extremal eigenvalue problem and developed a variational technique for solving such problems. We treat a slightly more general class of extremal eigenvalue problems and give sufficient conditions for a given function to be a solution. Our work on the strongest constrained column demonstrates a procedure for finding functions satisfying these conditions.
\end{abstract}

1. Introduction. Let $\lambda(\rho)$ denote the lowest eigenvalue of the boundary-value problem

$$
\begin{aligned}
y^{\prime \prime}+\lambda \rho(x) y & =0, \quad 0 \leq x \leq l, \\
\alpha_{1} y(0)-\beta_{1} y^{\prime}(0) & =0, \\
\alpha_{2} y(l)+\beta_{2} y^{\prime}(l) & =0
\end{aligned}
$$

where $\rho(x)>0$ and the $\alpha_{i}$ and $\beta_{i}$ are real numbers satisfying $\left|\alpha_{i}\right|+\left|\beta_{i}\right|>0, i=1,2$, and chosen in such a way that

$$
y^{\prime}(0) y(0)-y^{\prime}(l) y(l)+\int_{0}^{l}{y^{\prime 2}}^{2} d x>0
$$

for any nonzero $C^{2}$ function $y$ satisfying the boundary conditions in (1.1). This condition insures that all eigenvalues of the boundary value problem are $>0$. It is satisfied by many of the Sturm-Liouville problems commonly met in practice.

Let $M$ and $H>h \geq 0$ be given numbers and let $f(x, \rho)$ be a given real-valued continuous function defined on $[0, l] \times[h, H]$. In this paper, we shall study the extremal eigenvalue problem

$$
\text { maximize } \lambda(\rho) \text { subject to } \int_{0}^{l} f(x, \rho(x)) d x=M \quad h \leq \rho(x) \leq H .
$$

Several special cases of this problem have been solved by previous authors. In particular, the case where $f(x, \rho)=\rho^{-1 / 2}$ (this corresponds to the strongest column problem)

* Received August 7, 1975. 
was solved by Keller [1] with no constraints of the form $h \leq \rho(x) \leq H$ on $\rho$. This work was generalized to the case $f(x, \rho)=\rho^{n}, n<\frac{1}{2}$ by Tadjbakhsh and Keller [2].

Another important special case of (1.2) occurs when $f(x, \rho)=\rho$ and $\beta_{1}=\beta_{2}=0$. This problem was solved by M. G. Krein [3] with the constraints $h \leq \rho(x) \leq H$ present. Krein actually did much more than this. He determined both the maximum and minimum of each eigenvalue of (1.1) subject to the constraints in (1.2) with $f(x, \rho)=\rho$. His results were used to determine Lyapunov zones of stability for a parameter in Hill's equation. Problems similar to Krein's are studied in $[4,5,6]$.

2. The extremal eigenvalue problem. The constraints $h \leq \rho(x) \leq H$ imposed on the function $\rho$ in (1.1) are analogous to the ones we find in passing from classical problems in the calculus of variations to more general problems in optimal control theory where each admissible curve is required to lie in some designated region. We shall therefore use certain ideas from optimal control theory in dealing with the problem (1.2). Actually, all that is required is an elementary version of Pontryagin's maximum principle (which we state below as a minimum principle) and the reader is not assumed to have a background in optimal control theory.

The minimum principle that we require concerns problems of the form

$$
\operatorname{minimize} \int_{0}^{l} f_{0}(x, \rho(x)) d x
$$

$$
\text { subject to } \int_{0}^{l} f_{i}(x, \rho(x)) d x=M_{i} \quad(i=1,2, \cdots, N), \quad h \leq \rho(x) \leq H
$$

where $f_{0}, f_{1}, \cdots, f_{N}$ are given functions, each continuous on $[0, l] \times[h, H]$, and $M_{1}, \cdots$, $M_{N}$ are given constants.

Theorem 2.1 (Minimum Principle). If $\rho_{0}(x)$ is a solution of problem (2.1) then there exist constants (Lagrange multipliers) $\eta_{0} \geq 0, \eta_{1}, \cdots, \eta_{N}$, not all zero, such that

$$
\begin{aligned}
\min _{h \leq \rho \leq H}\left[\eta_{0} f_{0}(x, \rho)+\eta_{1} f_{1}(x, \rho)+\cdots+\eta_{N} f_{N}(x, \rho)\right] \\
\quad=\eta_{0} f_{0}\left(x, \rho_{0}(x)\right)+\eta_{1} f_{1}\left(x, \rho_{0}(x)\right)+\cdots+\eta_{N} f_{N}\left(x, \rho_{0}(x)\right)
\end{aligned}
$$

for each $x$ in $[0, l]$. Conversely, if there exist multipliers $\eta_{0}>0, \eta_{1}, \cdots, \eta_{N}$ and a function $\rho_{0}(x)$ such that $(2.2)$ holds, and if the conditions

$$
\int_{0}^{l} f_{i}\left(x, \rho_{0}(x)\right) d x=M_{i} \quad(i=1, \cdots, N), \quad h \leq \rho_{0}(x) \leq H
$$

are satisfied, then $\rho_{0}$ is a solution of (2.1).

This theorem appears in [7, page 215]. In our work below, once we have determined a function $\rho_{0}(x)$ satisfying the conditions of the theorem, we would like to know that this function is optimal. Therefore, we stress the sufficiency part of this theorem, and since the proof of this part of the theorem is extremely short we give it here.

Let $\rho_{0}(x)$ be given satisfying conditions (2.2) and (2.3) and assume that $\eta_{0}>0$. Let $\rho(x)$ be any other function satisfying (2.3). We shall show that

$$
\int_{0}^{l} f_{0}(x, \rho(x)) d x \geq \int_{0}^{l} f_{0}\left(x, \rho_{0}(x)\right) d x .
$$


We have

$$
\begin{aligned}
\eta_{0}\left[\int_{0}^{l} f_{0}(x, \rho(x)) d x-\int_{0}^{l}\right. & \left.f_{0}\left(x, \rho_{0}(x)\right) d x\right] \\
& =\int_{0}^{l}\left[\sum_{i=0}^{N} \eta_{i} f_{i}(x, \rho(x))\right] d x-\int_{0}^{l}\left[\sum_{i=0}^{N} \eta_{i} f_{i}\left(x, \rho_{0}(x)\right)\right] d x \geq 0
\end{aligned}
$$

by (2.2). Since $\eta_{0}>0$, (2.4) holds.

Our problem (1.2) is clearly not one of the form (2.1). To see how Theorem 2.1 applies to problem (1.2) we need Theorem 2.2 below. This theorem gives a sufficient condition for a function $\rho_{0}(x)$ to be a solution of problem (1.2). Having proved the theorem we can therefore solve problem (1.2) by constructing a function satisfying the conditions of the theorem. This will be the approach we shall take.

Theorem 2.2. Let $\rho_{0}(x)$ be a function satisfying

$$
\int_{0}^{l} f\left(x, \rho_{0}(x)\right) d x=M, \quad h \leq \rho_{0}(x) \leq H
$$

and let $y_{0}(x)$ be any eigenfunction of (1.1) (with $\rho=\rho_{0}$ ) corresponding to the lowest eigenvalue $\lambda\left(\rho_{0}\right)$. Then if

$$
\int_{0}^{l} y_{0}{ }^{2}(x) \rho_{0}(x) d x \leq \int_{0}^{l} y_{0}{ }^{2}(x) \rho(x) d x
$$

for every function $\rho(x)$ satisfying (2.5), $\rho_{0}(x)$ is a solution of (1.2).

Proof. Let $\rho(x)$ be any function satisfying (2.5). For simplicity assume that $\beta_{1} \neq 0$ and $\beta_{2} \neq 0$ in (1.1). We then have

$$
\lambda(\rho)=\min \frac{\left(\alpha_{1} / \beta_{1}\right) y^{2}(0)+\left(\alpha_{2} / \beta_{2}\right) y^{2}(l)+\int_{0}^{l} y^{\prime 2} d x}{\int_{0}^{l} \rho(x) y^{2} d x}
$$

where the minimization is taken over all functions $y$ having an absolutely continuous first derivative on $[0, l]$. It follows that

$$
\begin{aligned}
\lambda(\rho) & \leq \frac{\left(\alpha_{1} / \beta_{1}\right) y_{0}{ }^{2}(0)+\left(\alpha_{2} / \beta_{2}\right) y_{0}{ }^{2}(l)+\int_{0}^{l}{y_{0}}^{\prime 2} d x}{\int_{0}^{l} \rho(x) y_{0}{ }^{2} d x} \\
& \leq \frac{\left(\alpha_{1} / \beta_{1}\right) y_{0}{ }^{2}(0)+\left(\alpha_{2} / \beta_{2}\right) y_{0}{ }^{2}(l)+\int_{0}^{l}{y_{0}}^{\prime 2} d x}{\int_{0}^{l} \rho_{0}(x) y_{0}{ }^{2} d x} \\
& =\lambda\left(\rho_{0}\right) .
\end{aligned}
$$

This shows that $\rho_{0}$ is a solution of (1.2). The proof is similar if $\beta_{1}$ or $\beta_{2}$ is zero. In this case $\lambda(\rho)$ is still the minimum of the ratio of two quadratic forms over a certain class of functions and the proof is essentially unchanged. 
Another way of stating Theorem 2.2 is to say that if $\rho_{0}(x)$ is a solution of the problem

$$
\text { minimize } \int_{0}^{l} y_{0}{ }^{2}(x) \rho(x) d x \text { subject to } \int_{0}^{l} f(x, \rho(x)) d x=M, h \leq \rho(x) \leq H
$$

then $\rho_{0}(x)$ is a solution of $(1.2)$.

Problem (2.7) is one of the form (2.1) to which the minimum principle can be applied. Of course the eigenfunction $y_{0}(x)$ is not known but this will cause us no difficulty in applying the minimum principle. It turns out that $y_{0}(x)$ and $\rho_{0}(x)$ can be determined simultaneously.

By applying Theorem 2.1 to problem (2.7) we obtain the following result.

Theorem 2.3. In order for $\rho_{0}(x)$ to be a solution of problem (1.2) it is sufficient that

$$
\int_{0}^{l} f\left(x, \rho_{0}(x)\right) d x=M, \quad h \leq \rho_{0}(x) \leq H,
$$

and that there exist constants $\eta_{0}>0, \eta$ such that

$$
\min _{h \leq \rho \leq H}\left[\eta_{0} y_{0}^{2}(x) \rho+\eta f(x, \rho)\right]=\eta_{0} y_{0}^{2}(x) \rho_{0}(x)+\eta f\left(x, \rho_{0}(x)\right)
$$

for almost all $x$ in $[0, l]$, where $y_{0}(x)$ is an eigenfunction corresponding to the lowest eigenvalue of the boundary-value problem

$$
\begin{gathered}
y^{\prime \prime}+\lambda \rho_{0}(x) y=0, \\
\alpha_{1} y(0)-\beta_{1} y^{\prime}(0)=0, \quad \alpha_{2} y(l)+\beta_{2} y^{\prime}(l)=0 .
\end{gathered}
$$

One way of attempting to satisfy the conditions of this theorem is to select some convenient positive value of $\eta_{0}$, say $\eta_{0}=1$, and then define $\rho(x, y, \eta)$, for each $x \in[0, l]$ and for each real pair $(\eta, y)$, to be the solution of the problem

$$
\min _{h \leq \rho \leq H}\left[\eta_{0} y^{2} \rho+\eta f(x, \rho)\right] .
$$

Here $y$ and $\eta$ are to be treated as unknown parameters. When no confusion can arise we shall not indicate the dependence of $\rho$ on $y$ (or on $y$ and $\eta$ ) and shall simply write $\rho(x, \eta)$ (or $\rho(x))$ in place of $\rho(x, y, \eta)$. Having determined $\rho(x, y, \eta)$ we solve the nonlinear initial value problem

$$
\begin{gathered}
y^{\prime \prime}+\lambda \rho(x, \eta, y) y=0, \quad 0 \leq x \leq l, \\
y(0)=\beta_{1}, \quad y^{\prime}(0)=\alpha_{1} .
\end{gathered}
$$

with $\lambda$ and $\eta$ treated as parameters. (Note that $\alpha_{1} y(0)-\beta_{1} y^{\prime}(0)=0$.) Let $y(x, \lambda, \eta)$ denote the solution of this problem. Whenever no confusion can arise we shall simply write $y(x, \eta)$ or $y(x)$ in place of $y(x, \eta, \lambda)$. Now consider the nonlinear equations

$$
\begin{gathered}
\alpha_{2} y(l, \eta, \lambda)+\beta_{2} y^{\prime}(l, \eta, \lambda)=0, \\
\int_{0}^{l} f(x, \rho(x, \eta)) d x=M .
\end{gathered}
$$

(Clearly $\rho(x, \eta)$ depends on $\lambda$ since $y(x, \eta)$ does.) If we can determine $\lambda$ and $\eta$ such that these equations are satisfied and such that $\lambda$ is the lowest eigenvalue of the boundaryvalue problem (2.9) coupled with (2.10a), then we will have satisfied all the conditions 
of Theorem 2.3 with $\rho_{0}(x)=\rho(x, \eta)$ and $y_{0}(x)=y(x, \eta) . \rho_{0}(x)$, determined in this way, will therefore be a solution of problem 1.2.

In the remaining two sections of the paper, we describe how the procedure we have outlined can actually be carried out in particular instances. In particular, we solve the modified strongest column problem in Sec. 4.

3. Isoperimetric eigenvalue inequalities. The examples solved in this section demonstrate that, at least in certain cases, our procedure is easy to apply. They also serve to increase our understanding of the procedure.

Consider the problem

$$
\text { maximize } \lambda(\rho) \text { subject to } \int_{0}^{l} \rho^{n}(x) d x=M, \quad 0 \leq \rho(x) \leq H
$$

where $n, H$, and $M$ are given positive numbers satisfying $n \geq 1, M<l H^{n}$, and $\lambda(\rho)$ is the lowest eigenvalue of the boundary-value problem

$$
\begin{gathered}
y^{\prime \prime}+\lambda \rho(x) y=0, \quad 0 \leq x \leq l, \\
\alpha_{1} y(0)-y^{\prime}(0)=0, \quad \alpha_{2} y(l)+y^{\prime}(l)=0 .
\end{gathered}
$$

$\alpha_{1}$ and $\alpha_{2}$ are positive constants.

Problems similar to this one were solved in [2]. There the problems studied correspond to taking $H=\infty$ and $n<1$ in (3.1). Problem (3.1) has no solution for $H=\infty$ and $n \geq 1$. In fact, it can be seen from our work below that the maximum in (3.1) tends to $\infty$ as $H$ tends to $\infty$. The constraint $h \leq \rho(x) \leq H$ imposed on the functions $\rho$ admitted in (1.2) therefore has the desirable effect of establishing a solution in problems that otherwise would have none. This type of constraint is also desirable in problems such as the one involving column design to be described in Sec. 4. Without a lower bound on the thickness of admissible columns, the optimal ones would taper to a point at certain places along the length of the columns. Such columns are shown in [2] but probably would never be used in an actual structure.

We shall solve (3.1) by finding functions $\rho_{0}$ and $y_{0}$ satisfying the conditions of Theorem 2.3 for this problem. For convenience we take $\eta_{0}=n$ in (2.8). Then, in order to satisfy the conditions of Theorem 2.3 in the present case, we must choose $\rho_{0}(x)$ such that

$$
\int_{0}^{l} \rho_{0}{ }^{n}(x) d x=M, \quad 0 \leq \rho_{0}(x) \leq H,
$$

and such that

$$
\min _{0 \leq \rho \leq H}\left[n y^{2}(x) \rho+\eta \rho^{n}\right]=n y^{2}(x) \rho_{0}(x)+\eta \rho_{0}{ }^{n}(x)
$$

for almost all $0 \leq x \leq l$ and for some constant $\eta$.

Since $y(x)$ is an eigenfunction of (3.2) corresponding to the lowest eigenvalue, and since $\alpha_{1}>0, \alpha_{2}>0, y(x)$ can have no zeros on $[0, l]$. Thus, $y^{2}(x)>0$ for $0 \leq x \leq l$. From this it follows that if $\eta \geq 0$ in (3.3), the minimum there would be attained uniquely at $\rho=0$. But since $M>0$ we cannot have $\rho_{0}(x) \equiv 0$. We must therefore take $\eta<0$ in (3.3) if our method is to succeed. We do this, and in order to simplify the notation 
we replace $\eta$ by $-\eta$ and assume that $\eta>0$. (3.3) then requires that

$$
\min _{0 \leq \rho \leq H}\left[n y^{2}(x) \rho-\eta \rho^{n}\right]=n y^{2}(x) \rho_{0}(x)-\eta \rho_{0}{ }^{n}(x)
$$

for almost all $0 \leq x \leq l$ and some $\eta>0$.

For each $0 \leq x \leq l$, the function $n y^{2}(x) \rho-\eta \rho^{n}$ is concave in $\rho$. The minimum in (3.4) therefore occurs either at $\rho=0$ or $\rho=H$. Comparing the values of $n y^{2}(x) \rho-\eta \rho^{n}$ at these two points, we see that

$$
\begin{array}{rlll}
\rho_{0}(x) & =H & \text { if } & y^{2}(x)<\eta H^{n-1} / n \\
& =0 & \text { if } & y^{2}(x) \geq \eta H^{n-1} / n
\end{array}
$$

satisfies (3.4) and we shall choose $\rho_{0}(x)$ in this way.

Assume that $y^{2}(x)<\eta H^{n-1} / n$ for $x$ sufficiently close to 0 . It is easily verified that the converse leads to negative results. Thus, if our problem has a solution, it must satisfy $\rho_{0}(x)=H$ for $x$ sufficiently close to 0 . For these values of $x$ the differential equation in (3.2) is simply

$$
y^{\prime \prime}+\lambda H y=0 .
$$

This shows that near $x=0$ the point $\left(y(x), y^{\prime}(x)\right)$ is moving along an ellipse of the form

$$
{y^{\prime 2}}^{2}+\lambda H y^{2}=\text { constant }
$$

in $y, y^{\prime}$ space. Since eigenfunctions are unique only up to a scalar factor, we may assume that $y(0)$ and $y^{\prime}(0)$ are $>0$. Thus near $x=0 y(x)$ is increasing. Since we cannot have $\rho_{0}(x)=H$ for all $x$ in $[0, l]$ because of our assumption that

$$
\int_{0}^{l} \rho^{n}(x) d x=M<l H^{n}
$$

there will come a time $0<x_{1}<l$ when the condition

$$
y^{2}\left(x_{1}\right)=\eta H^{n-1} / n
$$

is satisfied. Assume that $y^{2}(x) \geq \eta H^{n-1} / n$ for $x$ in some interval $\left[x_{1}, x_{2}\right], x_{1}<x_{2} \leq l$, where $x_{2}$ has been chosen as large as possible. Then by (3.5) we can take $\rho_{0}(x)=0$ for $x_{1} \leq x \leq x_{2}$.

It must be the case that $y(x)$ is decreasing at $x=x_{2}$. That is, we must have $y^{\prime}\left(x_{2}\right) \leq 0$. For if we had $y^{\prime}\left(x_{2}\right)>0$ then the condition $y(x)>\eta H^{n-1} / n$ would be satisfied in some neighborhood of $x_{2}$, contradicting the fact that $x_{2}$ was chosen as large as possible.

Since $y(x) \neq 0$ for $0 \leq x \leq l$ we have

$$
y^{\prime \prime}(x)=-\lambda \rho_{0}(x) y(x) \leq 0
$$

on $[0, l]$. This implies that $y^{\prime}(x)$ is nonincreasing on $[0, l]$. In particular, since $y^{\prime}\left(x_{2}\right) \leq 0$ we have $y^{\prime}(x) \leq 0$ for $x_{2} \leq x \leq l$. It follows that $y(x)$ is nonincreasing on $\left[x_{2}, l\right]$. This implies that $y(x)<\eta H^{n-1} / n$ on $\left(x_{2}, l\right]$ and we therefore have

$$
\begin{aligned}
\rho_{0}(x) & =H, & & 0 \leq x<x_{1} \\
& =0, & & x_{1} \leq x \leq x_{2} \\
& =H, & & x_{2}<x \leq l .
\end{aligned}
$$


Since $\int_{0}^{l} \rho_{0}{ }^{n}(x) d x=M$ we must have

$$
x_{2}-x_{1}=l-\left(M / H^{n}\right) .
$$

We must also have

$$
\begin{aligned}
\lambda & =\frac{\alpha_{1} y^{2}(0)+\alpha_{2} y^{2}(l)+\int_{0}^{l} y^{\prime 2} d x}{\int_{0}^{l} \rho_{0} y^{2} d x} \\
& =\frac{\alpha_{1} y^{2}(0)+\alpha_{2} y^{2}(l)+\int_{0}^{x_{1}} y^{\prime 2} d x+\int_{x_{1}}^{x_{2}} y^{\prime 2} d x+\int_{x_{2}}^{l} y^{\prime 2} d x}{\int_{0}^{x_{2}} H y^{2} d x+\int_{x_{2}}^{l} H y^{2} d x} .
\end{aligned}
$$

The differential equation (3.2) implies that

$$
\int_{0}^{x_{1}} y^{\prime 2} d x=\int_{0}^{x_{1}} \lambda H y^{2} d x+y\left(x_{1}\right) y^{\prime}\left(x_{1}\right)-\alpha_{1} y^{2}(0)
$$

and

$$
\int_{x_{2}}^{l} y^{\prime 2} d x=\int_{x_{2}}^{l} \lambda H y^{2} d x-\alpha_{2} y^{2}(l)-y\left(x_{2}\right) y^{\prime}\left(x_{2}\right)
$$

It follows that

$$
\lambda=\frac{\lambda \int_{0}^{x_{1}} H y^{2} d x+\lambda \int_{x_{2}}^{l} H y^{2} d x+\int_{x_{2}}^{x_{2}} y^{\prime 2} d x+\left(\frac{\eta H^{n-1}}{n}\right)\left(y^{\prime}\left(x_{1}\right)-y^{\prime}\left(x_{2}\right)\right)}{\int_{0}^{x_{1}} H y^{2} d x+\int_{x_{2}}^{l} H y^{2} d x} .
$$

This equation implies that

$$
\left(\frac{\eta H^{n-1}}{n}\right)^{1 / 2}\left(y^{\prime}\left(x_{1}\right)-y^{\prime}\left(x_{2}\right)\right)+\int_{x_{1}}^{x_{2}}{y^{\prime 2}}^{2} d x=0,
$$

and, since $y^{\prime}\left(x_{1}\right) \geq 0$ and $y^{\prime}\left(x_{2}\right) \leq 0$, we must have $y^{\prime}(x)=0$ for $x_{1} \leq x \leq x_{2}$. The function $y$ is therefore such that the point $\left(y(x), y^{\prime}(x)\right)$ moves along the ellipse (3.7) in a clockwise direction, starting on the line $y^{\prime}=\alpha_{1} y$, to a point and time $x_{1}$ where

$$
y\left(x_{1}\right)=\left(\frac{\eta H^{n-1}}{n}\right)^{1 / 2} \text { and } y^{\prime}\left(x_{1}\right)=0 .
$$

The point $\left(y(x), y^{\prime}(x)\right)$ remains fixed at

$$
\left(\left(\frac{\eta H^{n-1}}{n}\right)^{1 / 2}, 0\right) \text { for } x_{1} \leq x \leq x_{2} .
$$

Then on the interval $\left[x_{2}, l\right]$ it continues along the ellipse (3.7) to the line $y^{\prime}=-\alpha_{2} y$.

We shall now determine expressions for $x_{1}$ and $x_{2}$ in terms of $\lambda$. To this end let $\theta(x)=$ $y^{\prime}(x) / y(x)$. Then for $x \in\left(0, x_{1}\right) \cup\left(x_{2}, l\right)$ we have $\theta^{\prime}=-\lambda H-\theta^{2}$. Moreover, $\theta\left(x_{1}\right)=$ $\theta\left(x_{2}\right)=0, \theta(0)=\alpha_{1}$, and $\theta(l)=\alpha_{2}$. It follows that

$$
\int_{0}^{\alpha_{1}} \frac{d \theta}{\lambda H+\theta^{2}}=\int_{0}^{x_{1}} d x
$$


or

$$
\frac{1}{(\lambda H)^{1 / 2}} \arctan \frac{\alpha_{1}}{(\lambda H)^{1 / 2}}=x_{1} .
$$

Similarly,

$$
\frac{1}{(\lambda H)^{1 / 2}} \arctan \frac{\alpha_{2}}{(\lambda H)^{1 / 2}}=l-x_{2} .
$$

We therefore have

$$
x_{2}-x_{1}=l-\frac{1}{(\lambda H)^{1 / 2}} \arctan \frac{\alpha_{2}}{(\lambda H)^{1 / \overline{2}}}-\frac{1}{(\lambda H)^{1 / 2}} \arctan \frac{\alpha_{1}}{(\lambda H)^{1 / 2}} .
$$

Combining this with (3.9) gives

$$
\arctan \frac{\alpha_{2}}{(\lambda H)^{1 / 2}}+\arctan \frac{\alpha_{1}}{(\lambda H)^{1 / 2}}=\frac{(\lambda H)^{1 / 2} M}{H^{n}} .
$$

Taking the tangent of each side of this equation gives the single equation

$$
\tan \frac{(\lambda H)^{1 / 2} M}{H^{n}}=\frac{(\lambda H)^{1 / 2}\left(\alpha_{1}+\alpha_{2}\right)}{\lambda H-\alpha_{1} \alpha_{2}}
$$

for $\lambda$. The desired $\lambda$ is the lowest eigenvalue of (3.2) for $\rho=\rho_{0}$. This $\lambda$ is therefore the smallest positive solution of (3.12). In summary we have

Theorem 3.1. Let $\Lambda$ denote the smallest positive solution of Eq. (3.12). Then the maximum in (3.1) is $\Lambda$ and is achieved by the function (3.8) with $x_{1}$ and $x_{2}$ given by (3.10) and (3.11) for $\lambda=\Lambda$.

Corollary. Let $\lambda_{1}$ denote the lowest eigenvalue of the boundary-value problem

$$
\begin{gathered}
y^{\prime \prime}+\lambda \rho(x) y=0, \quad 0 \leq x \leq l \\
y(0)=y(l)=0 .
\end{gathered}
$$

If $0 \leq \rho(x) \leq H$, and $\rho$ is not identically zero, then for any $n \geq 1$,

$$
\lambda_{1} \leq \pi H^{2 n-1}\left(\int_{0}^{l} \rho^{n}(x) d x\right)^{-2}
$$

Proof. This result may be obtained directly by computing the maximum of the lowest eigenvalue of (3.13) over all functions $\rho$ satisfying $0 \leq \rho(x) \leq H$ and for which the integral $\int_{0}^{l} \rho^{n}(x) d x$ has the value $M$ determined by the $\rho$ in (3.13). Alternatively, it may be obtained by treating (3.13) as a limiting case of (3.2) as $\alpha_{1}, \alpha_{2} \rightarrow \infty$. In this latter approach one obtains that

$$
\Lambda \rightarrow \frac{1}{H}\left(\frac{\pi H^{n}}{M}\right)^{2}=\pi^{2} H^{2 n-1}\left(\int_{0}^{l} \rho^{n}(x) d x\right)^{-2}
$$

as $\alpha_{1}, \alpha_{2} \rightarrow \infty$. The conclusion of the corollary now follows from the definition of $\Lambda$.

We remark that the bound (3.14) is sharp in the sense that equality holds for the function

$$
\begin{aligned}
\rho(x) & =H, & & 0 \leq x \leq x_{1} \\
& =0, & & x_{1}<x<x_{2} \\
& =H, & & x_{2} \leq x \leq l
\end{aligned}
$$


where $x_{1}=M / 2 H^{n}, x_{2}=l-\left(M / 2 H^{n}\right)$. These values of $x_{2}$ and $x_{1}$ are obtained by taking $\alpha_{1}=\alpha_{2}=+\infty$ in (3.10) and (3.11).

For $n=1$ the result (3.14) and our remark are contained in [3].

4. The shape of the strongest column. Let $l>0, V>0$ and $b>a>0$ be given numbers satisfying

$$
a l<V<b l .
$$

Consider an untwisted column of length $l$, volume $V$, and cross-sectional areas $A(x)$ satisfying

$$
a \leq A(x) \leq b, \quad 0 \leq x \leq l .
$$

Assume that all cross-sections are similar. Each such column will be called admissible. We wish to determine the strongest admissible column. This is the one whose critical buckling load is largest.

Let an admissible column be subjected to an axial load $P$ and let $w(x)$ denote the lateral deflection in the column caused by this load. Then, as described in [2], $w$ satisfies the differential equation

$$
\left(E I(x) w_{x x}\right)_{x x}+P w_{x x}=0, \quad 0 \leq x \leq l
$$

and some boundary conditions such as

$$
\begin{aligned}
w(0) & =w_{x}(0)=0 \\
w_{x}(l) & =P w_{x}(l)+\left(E I(l) w_{x x}(l)\right)_{x x}=\mathbf{0}
\end{aligned}
$$

or

$$
\begin{aligned}
& w(0)=w_{x}(0)=0 \\
& w(l)=w_{x x}(l)=0 .
\end{aligned}
$$

(There are other possibilities for the boundary conditions but we do not treat these here.) Conditions (4.4) correspond to a column clamped at $x=0$ and free at $x=l$. Conditions (4.5) correspond to a column clamped at $x=0$ and hinged at $x=l$.

In (4.3) $E$ is Young's modulus of the column material and $I(x)$ is the moment of inertia of a cross-section about a line through its centroid normal to the plane of the deflected column. $I(x)$ is related to the area $A(x)$ by $I(x)=\alpha A^{2}(x)$ where $\alpha$ is a proportionality constant determined by the shape of the cross-sections.

Let $y(x)$ and $\lambda$ be new variables defined by $y(x)=A^{2}(x) w_{x x}(x), \lambda=P / E \alpha$. (4.3) implies that

$$
y^{\prime \prime}+\lambda A^{-2}(x) y=0
$$

and the boundary conditions (4.4), (4.5) imply

$$
y^{\prime}(0)=0, \quad y(l)=0
$$

and

$$
y^{\prime}(0)+l^{-1} y(0)=0, \quad y(l)=0 .
$$

respectively. 
For a given admissible shape $A(x)$, let $\lambda$ denote the smallest value for which the equation (4.6), together with one set of the boundary conditions (4.7) or (4.8), has a nontrivial solution. Then $P=\lambda E \alpha$ is the critical buckling load of the column with shape $A(x)$. The shape of the strongest column is therefore the function $A(x)$ which makes $P$ as large as possible subject to the constraints

$$
\int_{0}^{l} A(x) d x=V, \quad a \leq A(x) \leq b .
$$

The first constraint fixes the volume of the column and the second restricts the thickness of the column.

To be specific we now restrict our attention to admissible columus which are clamped at $x=0$ and free at $x=l$. The analysis is similar in the clamped-hinged case. In the clamped-free case the relevant boundary conditions are given by (4.7). Let $\rho(x)=A^{-2}(x)$ and let $\lambda(\rho)$ denote the lowest eigenvalue of the boundary-value problem

$$
\begin{gathered}
y^{\prime \prime}+\lambda \rho(x) y=0, \quad 0 \leq x \leq l \\
y^{\prime}(0)=0, \quad y(l)=0 .
\end{gathered}
$$

The problem of determining the shape of the strongest column is then to

$$
\text { maximize } \lambda(\rho) \text { subject to } \int_{0}^{l} \rho^{-1 / 2}(x) d x=V, \quad h \leq \rho(x) \leq H,
$$

where $h=b^{-2}$ and $H=a^{-2}$.

We begin the solution of this problem by applying the results of Sec. 2 directly. In (2.8) we take, for convenience, $\eta_{0}=\frac{1}{2}$. We then define $\rho(x, \eta)$ for a fixed $\eta$, and for each $x \in[0, l]$, to be the solution of the problem

$$
\min _{h \leq \rho \leq H}\left[\frac{1}{2} y^{2}(x) \rho+\eta \rho^{-1 / 2}\right]
$$

where $y(x)=y(x, \lambda)$ is the solution of the initial-value problem

$$
y^{\prime \prime}+\lambda \rho(x, \eta) y=0, \quad y^{\prime}(0)=0, \quad y(0)=1,
$$

with $\lambda>0$ treated as an unknown parameter.

If we can choose $\eta$ and $\lambda$ such that

$$
\int_{0}^{l} \rho^{-1 / 2}(x, \eta) d x=V
$$

and

$$
y(l, \lambda)=0
$$

and such that $\lambda$ is the lowest eigenvalue of the boundary-value problem

$$
y^{\prime \prime}+\lambda \rho(x, \eta) y=0, \quad y^{\prime}(0)=0, \quad y(l)=0,
$$

then $\rho(x, \eta)$ will satisfy all the conditions of Theorem 2.3 and will therefore be a solution of problem (4.11).

Lemma 4.1. In order for $\rho(x, \eta)$ to satisfy conditions (4.12), (4.13), and (4.14) we must have $0<\eta<H^{3 / 2}$.

The proof is fairly simple and we shall omit it. 
We now begin our search for $\eta$ in the range $0<\eta<H^{3 / 2}$ and $\lambda>0$ satisfying conditions (4.12), (4.13), and (4.14). From (4.12) we deduce, reasoning as in the previous paragraph, that

$$
\begin{aligned}
\rho(x, \eta) & =h \quad \text { if } \quad \eta^{2 / 3}(y(x))^{-4 / 3} \leq h \\
& =\eta^{2 / 3}(y(x))^{-4 / 3} \text { if } h \leq \eta^{2 / 3}(y(x))^{-4 / 3} \leq H \\
& =H \quad \text { if } \quad \eta^{2 / 3}(y(x))^{-4 / 3} \geq H
\end{aligned}
$$

for $0 \leq x \leq l$ and $0<\eta<H^{3 / 2}$.

There are two cases to distinguish. First, let us assume that $h \leq \eta^{2 / 3}<H$. This is the less complicated case and we shall treat it first.

Near $x=0$ it follows from (4.15), together with the fact that $y(0)=1$, that

$$
\rho(x, \eta)=\eta^{2 / 3}(y(x))^{-4 / 3}
$$

and the differential equation in (4.13) becomes

$$
y^{\prime \prime}+\lambda \eta^{2 / 3} y^{-1 / 3}=0 .
$$

It is convenient to view the solution in the phase, or $y, y^{\prime}$, plane (see Fig. 1). Near $x=0$ the solution satisfies

$$
\frac{d}{d x}\left(\frac{1}{2} y^{\prime 2}+\frac{3}{2} \lambda \eta^{2 / 3} y^{2 / 3}\right)=0
$$

Since $y^{\prime}(0) L=0$ and $y(0)=1$, initially the solution is traveling along the curve

$$
\frac{1}{2} y^{\prime 2}+\frac{3}{2} \lambda \eta^{2 / 3} y^{2 / 3}=\frac{3}{2} \lambda \eta^{2 / 3}
$$

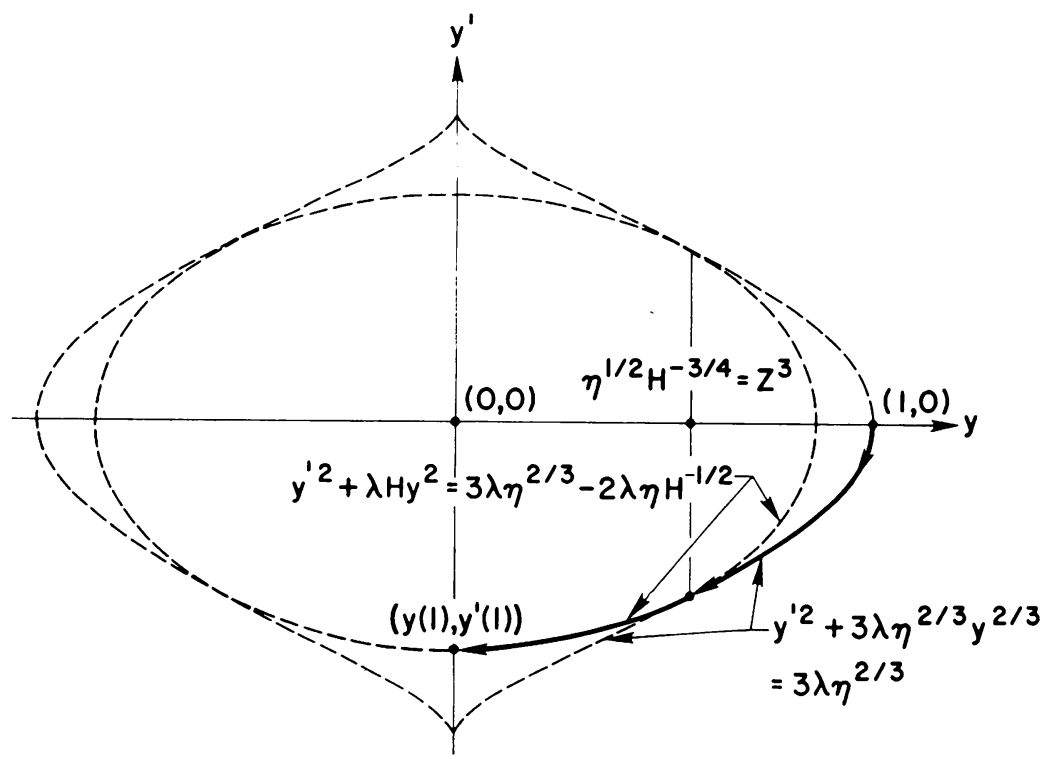

FIg. 1. The path of the solution is indicated by arrows. This is the case where $h \leqq \eta^{2 / 3}<H$. 
in phase space. Since $y(x)$ is decreasing and $y(l)=0$, there will come a time $0<x_{1}<l$ when

$$
\eta^{2 / 3}\left(y\left(x_{1}\right)\right)^{-4 / 3}=H
$$

We will then have

$$
\rho(x, \eta)=H
$$

for $x_{1} \leq x \leq l$. To determine an expression for $x_{1}$ we introduce a new function $\theta(x)$ defined on $\left[0, x_{1}\right]$ by the equation

$$
\sin ^{3} \theta(x)=y(x) .
$$

Substituting this into (4.16), we find that $\theta(x)$ must satisfy the differential equation

$$
\sin ^{2} \theta(d \theta / d x)=-\eta^{1 / 3} \sqrt{\frac{\lambda}{3}}, \quad \theta(0)=\pi / 2 .
$$

By separating variables in this equation and integrating we obtain

$$
\int_{\pi / 2}^{\theta\left(x_{1}\right)} \sin ^{2} \theta d \theta=-\eta^{1 / 3} \sqrt{\frac{\lambda}{3}} x_{1}
$$

or

$$
\frac{\theta\left(x_{1}\right)}{2}-\frac{\sin 2 \theta\left(x_{1}\right)}{4}-\frac{\pi}{4}=-\eta^{1 / 3} \sqrt{\frac{\lambda}{3}} x_{1} .
$$

This equation can be written as

$$
\frac{1}{2}\left[\theta\left(x_{1}\right)-\sin \theta\left(x_{1}\right)\left(1-\sin ^{2} \theta\left(x_{1}\right)\right)^{1 / 2}-\frac{\pi}{2}\right]=-\eta^{1 / 3} \sqrt{\frac{\lambda}{3}} x_{1} .
$$

Now by (4.17) we have

$$
\sin ^{3} \theta\left(x_{1}\right)=y\left(x_{1}\right)=\eta^{1 / 2} H^{-3 / 4} .
$$

Thus

$$
\sin \theta\left(x_{1}\right)=\eta^{1 / 6} H^{-1 / 4} .
$$

Substituting this into (4.18) we obtain

$$
x_{1}=\eta^{-1 / 3} \sqrt{\frac{3}{4 \lambda}}\left\{\frac{\pi}{2}+\eta^{1 / 6} H^{-1 / 4}\left(1-\eta^{1 / 3} H^{-1 / 2}\right)^{1 / 2}-\arcsin \left(\eta^{1 / 6} H^{-1 / 4}\right)\right\} .
$$

On the interval $\left[x_{1}, l\right]$ we have $\rho(x, \eta) \equiv H$ and the differential equation in (4.13) is simply

$$
y^{\prime \prime}+\lambda H y=0 .
$$

Along a solution of this equation we have

$$
\frac{d}{d x}\left[y^{\prime 2}+\lambda H y^{2}\right]=0 .
$$

Recall that $y\left(x_{1}\right)=\eta^{1 / 2} H^{-3 / 4}$. Substituting this into (4.16) gives

$$
y^{\prime 2}\left(x_{1}\right)=3 \lambda \eta^{2 / 3}-3 \lambda \eta H^{-1 / 2} \text {. }
$$


Thus

$$
y^{\prime 2}\left(x_{1}\right)+\lambda H y^{2}\left(x_{1}\right)=3 \lambda \eta^{2 / 3}-2 \lambda \eta H^{-1 / 2}
$$

and it therefore follows from (4.20) that, in phase space, the solution of (4.13) moves along the ellipse

$$
y^{\prime 2}+\lambda H y^{2}=3 \lambda \eta^{2 / 3}-2 \lambda \eta H^{-1 / 2}, \quad x_{1} \leq x \leq l .
$$

Since $y(l)=0$ we must have

$$
y^{\prime}(l)=-\left(3 \lambda \eta^{2 / 3}-2 \lambda \eta H^{-1 / 2}\right)^{1 / 2} .
$$

It follows that

$$
y(x)=\left(\frac{3 \lambda \eta^{2 / 3}-2 \lambda \eta H^{-1 / 2}}{\lambda H}\right)^{1 / 2} \sin (\lambda H)^{1 / 2}(l-x)
$$

for $x_{1} \leq x \leq l$. From this, together with the condition $y\left(x_{1}\right)=\eta^{1 / 2} H^{-3 / 4}$, we deduce that

$$
x_{1}=l-\frac{1}{(\lambda H)^{1 / 2}} \arcsin \left\{\frac{\eta^{1 / 3} H^{-1 / 2}}{3-2 \eta^{1 / 3} H^{-1 / 2}}\right\}^{1 / 2} .
$$

Here and in (4.19) the multi-valued function arc sin is to be given its principal value in $[0, \pi / 2]$. This is to insure that $x_{1}$ corresponds to the first intersection of the curves (4.16) and (4.21) in the fourth quadrant of phase space. Note that the curves have other intersections corresponding to larger values of $x_{1}$.

Equating (4.22) to the value of $x_{1}$ given by (4.19), we obtain the equation

$$
\begin{aligned}
\eta^{-1 / 3} \sqrt{\frac{3}{4 \lambda}}\left\{\frac{\pi}{2}+\eta^{1 / 6} H^{-1 / 4}\left(1-\eta^{1 / 3} H^{-1 / 2}\right)^{1 / 2}-\arcsin \left(\eta^{1 / 6} H^{-1 / 4}\right)\right\} \\
+\frac{1}{(\lambda H)^{1 / 2}} \arcsin \left\{\frac{\eta^{1 / 3} H^{-1 / 2}}{3-2 \eta^{1 / 3} H^{-1 / 2}}\right\}^{1 / 2}=l
\end{aligned}
$$

for $\eta$ and $\lambda$. We can obtain a second equation for these variables from the relation

$$
\int_{0}^{l} \rho^{-1 / 2}(x, \eta) d x=V
$$

We have

$$
\begin{aligned}
\int_{0}^{l} \rho^{-1 / 2}(x, \eta) & d x=\int_{0}^{x_{1}} \eta^{-1 / 3} \sin ^{2} \theta(x) d x+H^{-1 / 2}\left(l-x_{1}\right) \\
= & \eta^{-1 / 3} \int_{\pi / 2}^{\theta\left(x_{1}\right)} \sin ^{2} \theta \frac{d x}{d \theta} d \theta+H^{-1 / 2}\left(l-x_{1}\right) \\
= & -\eta^{-2 / 3} \sqrt{\frac{3}{\lambda}} \int_{\pi / 2}^{\theta\left(x_{1}\right)} \sin ^{4} \theta d \theta+H^{-1 / 2}\left(l-x_{1}\right) \\
= & \left.\eta^{-2 / 3} \sqrt{\frac{3}{\lambda}} \frac{\sin ^{3} \theta \cos \theta}{4}\right|_{\pi / 2} ^{\theta\left(x_{1}\right)}-\eta^{-2 / 3} \sqrt{\frac{3}{\lambda}} \cdot \frac{3}{4} \int_{\pi / 2}^{\theta\left(x_{1}\right)} \sin ^{2} \theta d \theta+H^{-1 / 2}\left(l-x_{1}\right) .
\end{aligned}
$$

Simplifying this expression and making use of (4.22), Eq. (4.24) takes the form

$$
\begin{aligned}
& \eta^{-2 / 3} \sqrt{\frac{3}{\lambda}}\left\{\eta^{1 / 2} H^{-3 / 4}\left(1-\eta^{1 / 3} H^{-1 / 2}\right)^{1 / 2}-\frac{3}{8} \arcsin \left(\eta^{1 / 6} H^{-1 / 4}\right)+\frac{3}{8} \eta^{1 / 6} H^{-1 / 4}\right. \\
&\left.\cdot\left(1-\eta^{1 / 3} H^{-1 / 2}\right)^{1 / 2}+\frac{3 \pi}{16}\right\}+\frac{1}{H \sqrt{ } \lambda} \arcsin \left\{\frac{\eta^{1 / 3} H^{-1 / 2}}{3-2 \eta^{1 / 3} H^{-1 / 2}}\right\}^{1 / 2}=V .
\end{aligned}
$$


If we multiply Eq. (4.23) by $(\lambda H)^{1 / 2}$ and set $z=\eta^{1 / 6} H^{-1 / 4}$ we can rewrite (4.23) as

$$
z^{-2} \sqrt{\frac{3}{4}}\left\{\frac{\pi}{2}+z\left(1-z^{2}\right)^{1 / 2}-\arcsin (z)\right\}+\arcsin \left\{\frac{z^{2}}{3-2 z^{2}}\right\}^{1 / 2}=l(\lambda H)^{1 / 2} \text {. }
$$

Similarly, if we multiply Eq. (4.25) by $H \sqrt{ } \lambda$ we obtain the equation

$$
\begin{aligned}
z^{-4} \sqrt{ } 3\left\{\frac{3 \pi}{16}+z^{3}\left(1-z^{2}\right)^{1 / 2}-\frac{3}{8} \arcsin (z)\right. & \left.+\frac{3}{8} z\left(1-z^{2}\right)^{1 / 2}\right\} \\
& +\arcsin \left\{\frac{z^{2}}{3-2 z^{2}}\right\}^{1 / 2}=V H \sqrt{ } \lambda .
\end{aligned}
$$

Now if we divide Eq. (4.27) by Eq. (4.26) we obtain the single equation

$$
\begin{aligned}
& z^{-4} \sqrt{ } 3\left\{\frac{3 \pi}{16}+z^{3}\left(1-z^{2}\right)^{1 / 2}-\frac{3}{8} \arcsin (z)+\frac{3}{8} z\left(1-z^{2}\right)^{1 / 2}\right\}+\arcsin \left\{\frac{z^{2}}{3-2 z^{2}}\right\}^{1 / 2} \\
& z^{-2} \sqrt{\frac{3}{4}}\left\{\frac{\pi}{2}+z\left(1-z^{2}\right)^{1 / 2}-\arcsin (z)\right\}+\arcsin \left\{\frac{z^{2}}{3-2 z^{2}}\right\}^{1 / 2} \\
& =V H^{1 / 2} / l=V / a l
\end{aligned}
$$

for the unknown $z=\eta^{1 / 6} H^{-1 / 4}$.

Lemma 4.2. If the function arc sin is given its principal value in $[0, \pi / 2]$ for positive values of its argument, then Eq. (4.28) has a unique solution in the range $0<z<1$ for any choice of $V, a$, and $l$, satisfying $a l<V$.

The proof of this is fairly simple and we shall omit it.

We summarize and interpret the results obtained so far in the following theorem.

TheOREM 4.1. Let $z_{1}$ be the solution of Eq. (4.28) satisfying $0<z_{1}<1$. If $z_{1} \geq$ $(h / H)^{1 / 4}$ set $\eta=H^{3 / 2} z_{1}{ }^{6}$,

$$
\left.\lambda=\frac{z_{1}^{-4}}{H l^{2}}\left[\sqrt{\frac{3}{4}}\left\{\frac{\pi}{2}+z_{1}\left(1-z_{1}\right)^{2}\right)^{1 / 2}-\arcsin \left(z_{1}\right)\right\}+\arcsin \left\{\frac{z_{1}{ }^{2}}{3-2 z_{1}{ }^{2}}\right\}^{1 / 2}\right]^{2}
$$

and

$$
x_{1}=\eta^{-1 / 3} \sqrt{\frac{3}{4}}\left\{\frac{\pi}{2}+z_{1}\left(1-z_{1}^{2}\right)^{1 / 2}-\arcsin \left(z_{1}\right)\right\},
$$

where the multi-valued function arc sin is given its principal value in $[0, \pi / 2]$.

Then define $\rho(y)$ by

$$
\begin{aligned}
\rho(y) & =h \quad \text { if } \quad \eta^{2 / 3} y^{-4 / 3} \leq h \\
& =\eta^{2 / 3} y^{-4 / 3} \quad \text { if } \quad h \leq \eta^{2 / 3} y^{-4 / 3} \leq H \\
& =H \quad \text { if } \quad \eta^{2 / 3} y^{-4 / 3} \geq H
\end{aligned}
$$

and solve the initial value problem

$$
\begin{aligned}
y^{\prime \prime}+\lambda \rho(y) y & =0, & & 0 \leq x \leq l \\
y(0) & =1, & & y^{\prime}(0)=0
\end{aligned}
$$

as we did above. Let $y(x)$ denote the solution. Define the admissible shape $A(x)$ by 


$$
\begin{aligned}
A(x) & =\eta^{-1 / 3} y^{2 / 3}(x), \quad 0 \leq x \leq x_{1} \\
& =a, \quad x_{1} \leq x \leq l .
\end{aligned}
$$

Then $A(x)$ is the shape of the strongest column in the class of columns of length $l$, volume $V$, clamped at $x=0$ and free at $x=l$, and having cross-sectional areas between $a=H^{-1 / 2}$ and $b=h^{-1 / 2}$.

It should be observed that the conditions of this theorem will be satisfied if $h$ is sufficiently small relative to $H$. In particular, when there is no restriction on the maximum thickness of admissible columns (i.e., when $h=0$ ), the optimal shape is given by (4.29)

Suppose now that the solution $z_{1}$ of Eq. (4.28) in the region $0<z<1$ satisfies $z_{1}<$ $(h / H)^{1 / 4}$. This is the second case to distinguish in solving problem (4.11). In this case, the $\eta$ that belongs in (4.12) satisfies $0<\eta^{2 / 3}<h$. We leave the problem of determining equations for $\eta$ and $\lambda$ to the reader in this case.

In closing we wish to acknowledge the paper [8] by Taylor and Liu. They solve a column design problem which can be thought of as dual to the one we have studied here. Instead of fixing the weight of the column and maximizing the load they fix the load and minimize the weight. The striking feature of their work is that they impose a lower bound on the thickness of admissible columns. They solve the resulting constrained minimization problem using techniques from the calculus of variations. They use the model (4.3) of a column and assume that the weight of the column is proportional to $\int_{0}^{l} I(x) d x$. This, of course, is not true, but it may be a reasonable assumption from a practical point of view since it assumes the weight to be proportional to $\int_{0}^{n} A^{2}(x) d x$. This assumption is useful in proving that the column found in [8] minimizes $\int_{0}^{n} A^{2}(x) d x$. This proof makes strong use of the form of the design objective and does not apply to the more general extremal eigenvalue problems studied here.

Two other papers closely related to the work reported here have been called to our attention by a referee. The first [9] of these papers deals with determining the shape of the strongest circular arch (see also [10]). These authors consider inextensional buckling in their planes of uniformly loaded simply-supported circular arches, with opening angle $2 \alpha$ sufficiently large that anti-symmetrical buckling is critical. For a pictorial description of the problem see [9].

Let $R$ denote the circular radius of the arch and let $w$ denote the radial displacement of the arch's central line. Let $P$ denote the critical buckling load. Then $P$ is given by the Rayleigh quotient

$$
P=\int_{0}^{l} E I\left(w_{s s}+w / R^{2}\right)^{2} d s / \int_{0}^{l}\left[w_{s}{ }^{2}-(w / R)^{2}\right] d s
$$

and $w$ minimizes this quotient subject to

$$
w(0)=0, \quad E I w_{s s}(0)=0, \quad w(l)=0, \quad E I w_{s s}(l)=0 .
$$

$s$ denotes arc length measured along the central line of the arch, $E$ is Young's modulus of the arch material and $I$ is the moment of inertia of the arch and is related to area $A(s)$ of cross-sections by

$$
I / V l=(A l / V)^{n}, \quad V=\int_{0}^{l} A(s) d s,
$$

where $n=1,2$, or 3 . These values of $n$ correspond to light-core sandwiches of constant 
width and depth, solid cross-sections of fixed shape, and solid rectangular sections of constant width. The problem of optimal arch design is to minimize (4.30) with respect to $w$ and then to maximize with respect to $A$ subject to $\int_{0}^{l} A(s) d x=V$.

The Euler equation associated with (4.30) is

$$
\begin{array}{r}
\left(\left(\frac{A l}{V}\right)^{n}\left(w_{s s}+w / R^{2}\right)\right)_{s s}+\frac{1}{R^{2}}\left(\frac{A l}{V}\right)^{n}\left(w_{s s}+w / R^{2}\right)+\frac{P}{E V l}\left(w_{s s}+w / R^{2}\right)=0, \\
w(0)=0, \quad A(0) w_{s s}(0)=0, \quad w(l)=0, \quad A(l) w_{s s}(l)=0 .
\end{array}
$$

Let $\rho(s)=(A(s) l / V)^{-n}, y(s)=\rho(s)^{-1}\left(w_{s s}(s)+w(s) / R^{2}\right)$, and $\lambda=P / E V l$. We then have, by $(4.31)$,

$$
y^{\prime \prime}+\left(1 / R^{2}\right) y+\lambda \rho(s) y=0, \quad\left({ }^{\prime}=d / d s\right), \quad y(0)=y(l)=0 .
$$

The volume of the arch is

$$
V=\int_{0}^{l} A(s) d s=\frac{V}{l} \int_{0}^{l} \rho^{-1 / n}(s) d s .
$$

The problem of determining the strongest circular arch is equivalent to determining $\rho$ to maximize the lowest eigenvalue of (4.32) subject to the isoperimetric constraint (4.33). This problem is solved in [9] and [10]. The solution given in [9] uses an argument similar to the one used in [2]. If magnitude constraints are placed on the areas of cross-sections it is clear that the resulting problem can be solved by the procedure described in this paper.

The problem studied in [11] has to do with determining the optimal shape of a vibrating beam. A simply supported beam performing small harmonic vibrations in a plane is considered. Such a beam has an infinite number of natural frequencies. The problem is to determine the shape of the beam which makes the lowest frequency as large as possible. The modes of vibration of the beam satisfy the equation

$$
\left(E I w_{x x}\right)_{x x}-\omega^{2} \gamma A w=0
$$

where $w$ is the lateral deflection in the plane of bending, $x$ is the coordinate along the axis of the beam, $E$ is Young's modulus, $I$ the principal moment of inertia of the crosssection perpendicular to the plane of bending, $\omega$ the natural angular frequency, $\gamma$ the mass per unit volume, and $A$ the cross-sectional area. The length and volume of the beam are assumed to be fixed. For simply-supported beams we have the boundary conditions

$$
w(0)=0, \quad E I w_{x x}(0)=0, \quad w(l)=0, \quad E I w_{x x}(l)=0,
$$

where $l$ is the length of the beam.

The problem of determining the optimal beam is equivalent to determining the function $A(x)$ to maximize the lowest eigenvalue $\lambda=\omega^{2}$ of the boundary value problem (4.34)-(4.35) subject to the fixed volume constraint

$$
\int_{0}^{l} A(x) d x=V .
$$

Even though this problem bears some resemblance to the problems studied in [1] and [2], it is considerably more difficult. In the first place, the optimal eigenfunction satisfies a fourth-order nonlinear eigenvalue problem with singular boundary conditions. Unlike 
the analogous equation which comes up in [1] and [2], this equation cannot be solved in closed form. However, an iterative procedure for constructing the solution is described in [11]. This makes it possible to determine the solution numerically. The optimal area function $A(x)$ is an explicit function of the optimal eigenfunction and its second derivative.

The method we have developed for determining the shape of the strongest constrained column can be made to apply to the beam design problem when constraints of the form

$$
a \leq A(x) \leq b
$$

are placed on the areas of cross-sections. Of course, the actual implementation of the procedure would have to be done numerically. We shall not present the details of this here.

The author wishes to thank the referee for calling his attention to references $[9,10,11]$.

\section{REFERENCES}

[1] J. B. Keller, The shape of the strongest column, Arch. Rat. Mech. Anal. 5, 275-285 (1960)

[2] I. Tadjbakhsh and J. B. Keller, Strongest column and isoperimetric inequalities for eigenvalues, ASME J. Appl. Mech. E29, 159-164 (1962)

[3] M. G. Krein, On certain problems on the maximum and minimum of characteristic values and on Lyapunov on stability, Prikl. Mat. Meh. 15, 323-348 (1951) (Russian); English translation in Amer. Math. Soc. Translations, Series 2, 1, 163-187 (1955)

[4] P. Nowosad, Isoperimetric problems in algebras, Comm. Pure Appl. Math. 21, 401-465 (1968)

[5] P. Nowosad, On extremal problems related to eigenvalues of linear differential operators, $I$, MRC Technical Summary Report \#1016, Mathematics Research Center, University of Wisconsin, Madison September 1969

[6] S. Karlin, Some extremal problems for eigenvalues of certain matrix and integral operators, Advances in Mathematics 9, 93-136 (1972)

[7] M. R. Hestenes, Calculus of variations and optimal control theory, John Wiley and Sons, Inc., New York, 1966

[8] J. E. Taylor and C. Y. Liu, Optimal design of columns, AIAA J. 6, 1497-1502 (1968)

[9] B. Budiansky, J. C. Frauenthal and J. W. Hutchinson, On optimal arches, ASME J. Appl. Mech. E36, 880-882 (1964)

[10] C. H. Wu, The strongest circular arch--a perturbation solution, ASME J. Appl. Mech. E35, 476-580 (1968)

[11] F. I. Niordson, On the optimal design of a vibrating beam, Quart. Appl. Math. 23, 47-53 (1965) 